\title{
MODELAGEM TRIDIMENSIONAL DE SUPERFÍCIES PLANAS A PARTIR DE IMAGEM TOF
}

\author{
Model plane surfaces with a 3D data obtained with range image \\ ANDREY AUGUSTO ALVES DE OLIVEIRA \\ JORGE ANTONIO SILVA CENTENO \\ Universidade Federal do Paraná - UFPR \\ Curso de Pós-Graduação Em Ciências Geodésicas \\ Caixa Postal 19.001 - Centro Politécnico - J. américas \\ CEP: 81531-990 Curitiba - Paraná - Brasil \\ andreyaugusto6@hotmail.com; centeno@ufpr.br
}

\begin{abstract}
RESUMO
Neste artigo é apresentado um estudo para a modelagem tridimensional de superfícies utilizando imagens obtidas com uma câmara TOF. O sistema utilizado é a câmara PMD CamCube 2.0, que calcula a distância de objetos utilizando o princípio TOF (Time-Of-Flight). Inicialmente, a câmara foi calibrada, o que possibilitou a correção geométrica da imagem a partir de Parâmetros de Orientação Interior (POI). Outro fator que afeta a qualidade dos dados é a presença de ruído em função da iluminação exterior ao sistema, que pode ser controlada variando o tempo de integração na captação da imagem. Posteriormente realizou-se a segmentação da imagem TOF para identificar as superfícies planas do objeto estudado. A solução encontrada utiliza o cálculo dos autovalores locais em superfícies, em pequenas janelas móveis. Com isto, gera-se uma nova imagem com bordas evidenciadas. A nova imagem é segmentada através do algoritmo de deslocamento pela média, com o objetivo de obter superfícies planas do objeto de interesse. Os píxeis da imagem segmentada são então projetados ortogonalmente, gerando uma nuvem de pontos com coordenadas tridimensionais das superfícies. Finalmente, as dimensões do objeto são comparadas com medidas de referência, sendo verificado que a diferença na medida tridimensional obtida é de aproximadamente 2 centímetros.

Palavras-chave: Imagem TOF; Modelagem Tridimensional de Superfícies; Autovalores; Autovetores; Segmentação de Imagens; Algoritmo de Deslocamento pela Média; Projeção Ortogonal de Pontos 3D.
\end{abstract}


ABSTRACT

In this paper we present a work that aims at studying the quality of $3 \mathrm{D}$ data obtained with a range camera and assessing its potential to model planes. The device, the PMD CamCube 2.0, computes the distance to the objects using the Time-Of-Flight (TOF) principle. At first, the equipment was calibrated. The Interior Orientation Parameters (IOP) allows reducing geometric distortion in the images. Another factor that influences the quality of data is the presence of noise introduced by illumination conditions, which can be controlled by varying the integration time of the light source. After determining the best integration time and interior orientation parameters, the device was used to capture data of an object with flat surfaces. The dataset was used to compute the 3D coordinates of each point and then the dataset was segmented, in order to identify the flat surfaces of the object. The segmentation solution is based on the computation of the local eigenvalues, using a small moving window, to generate a new image where borders are highlighted. This new image is segmented using the mean-shift approach to delineate the planes of interest. Finally, the dimensions of the object are compared to reference values. The comparison showed errors around $2 \mathrm{~cm}$, within the expected range. The object can then be modeled with the 3D points cloud.

Keywords: Range Image; Three-dimensional Modeling of Objects Surfaces; Eigenvalues; Eigenvectors; Images Segmentation; Mean-Shift; 3D Points Orthogonal Projection.

\section{INTRODUÇÃO}

Nas últimas décadas foram desenvolvidos diferentes equipamentos para a coleta de dados tridimensionais. Um dos mais recentes avanços são as câmaras TOF, que são dispositivos capazes de medir a distância entre o sensor e a superfície dos objetos utilizando o princípio TOF. Estes dispositivos têm atraído a atenção de diferentes campos que se interessam por medidas e coordenadas tridimensionais de objetos, tais como a Visão Computacional e a Engenharia de Automação. As câmaras TOF possibilitam a obtenção de 25 a 30 quadros por segundo (Frames Per Secund - fps). Com esta frequência de aquisição de imagens é possível registrar o movimento de objetos na cena, além de uma grande quantidade de informação 3D sobre os mesmos.

No entanto, estes dados devem ter sua qualidade avaliada, pois depende de fatores externos ao sistema, como: iluminação ambiente, distâncias envolvidas, orientações e refletividade entre as superfícies dos objetos e o sensor. As influências causadas por erros sistemáticos, assim como pela presença de ruído, devem ser observadas para cada tipo de aplicação da câmara TOF e de suas imagens (MAY et al., 2008).

Um exemplo de câmara TOF é a PMD CamCube 2.0. Esta utiliza um sensor PMD (Photonic Mixer Device), que possibilita armazenar informação de distância dos píxeis da imagem de intensidade captada.

Bol. Ciênc. Geod., sec. Artigos, Curitiba, v. 17, nº 3, p.361-378, jul-set, 2011. 
Algumas aplicações das imagens TOF já foram realizadas na prevenção de acidentes entre automóveis e segurança de pedestres (RINGBECK, 2007), Visão Computacional (WIEDEMANN et al., 2008), mapeamentos tridimensionais de superfícies em ambientes fechados para a movimentação robótica (MAY et al., 2008), Computação Gráfica (KOLB et al., 2009; KOCH et al., 2009), e análise de deformações (JAMTSHO, 2010).

\section{REVISÃO BIBLIOGRÁFICA}

\subsection{Imagem TOF}

A imagem TOF, também conhecida por range image ou imagem de distância, proporciona a análise da profundidade dos objetos na cena, além da informação de intensidade com que o sinal luminoso emitido foi refletido pelas superfícies do objeto imageado e captado pelo sensor. A Figura 1a ilustra o arquivo com as intensidades da imagem TOF e a Figura $1 \mathrm{~b}$ corresponde ao arquivo com as distâncias dos píxeis.

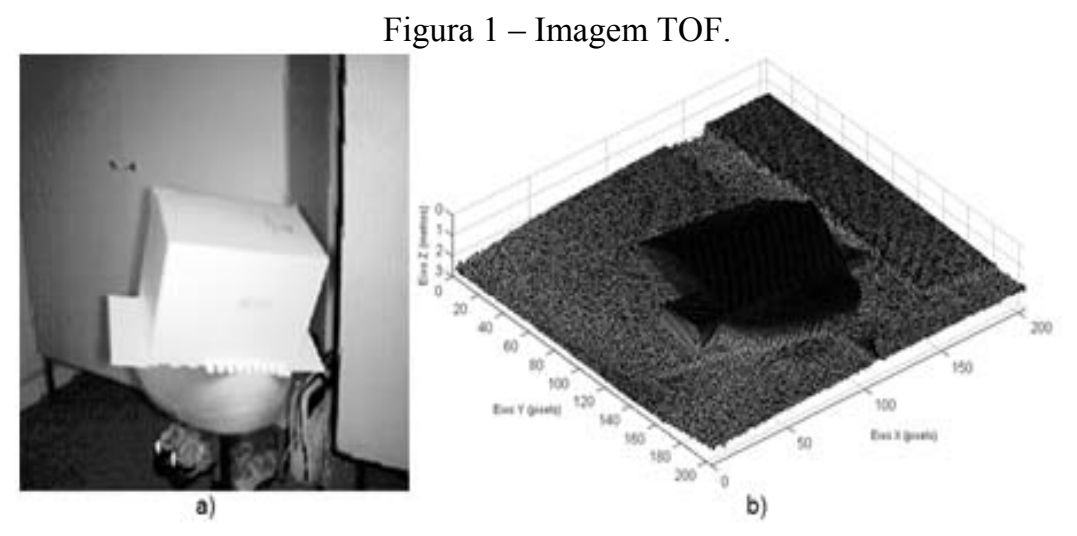

No sistema PMD a distância entre o sensor e o alvo é medida através do princípio TOF com o método da diferença de fase. Para isto utiliza-se uma Função de Correlação (FC) entre o sinal emitido e o sinal recebido. A iluminação é feita através de díodos emissores de luz LED, tendo-se a vantagem que a FC pode ser obtida com o sensor PMD, a partir da forma senoidal do sinal recebido (RAPP, 2007).

A análise da FC entre o sinal emitido e recebido pelo sensor é realizada através do método da diferença de fase, ilustrado com a Figura 2. 
Figura 2 - FC utilizada no método da diferença de fase.

Fonte: Adaptado de LANGE (2000).

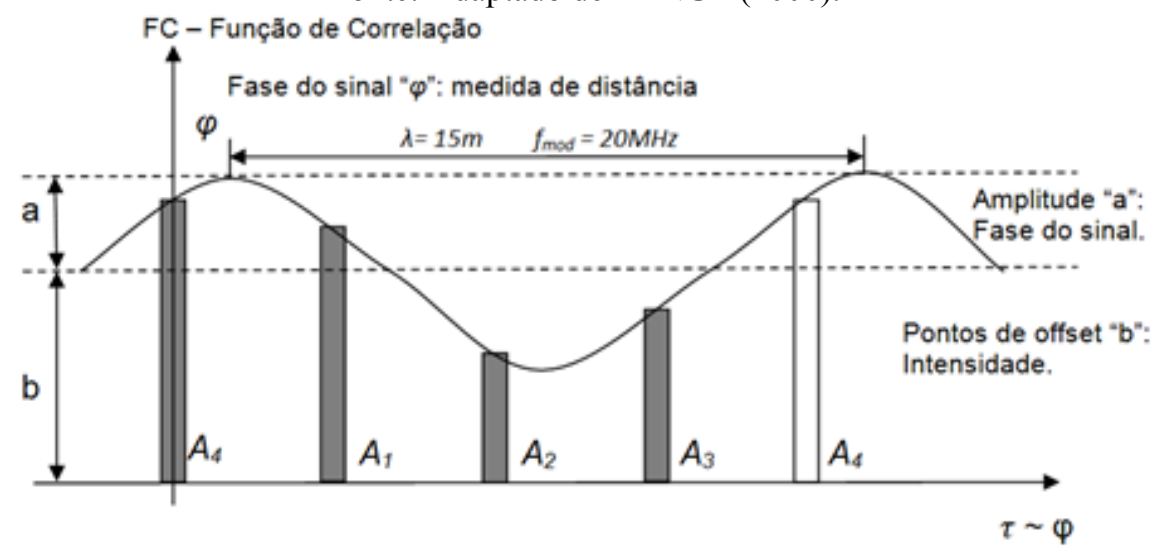

A FC permite calcular a distância entre o alvo e a câmara utilizando quatro amostras $\left(\mathrm{A}_{1}, \mathrm{~A}_{2}, \mathrm{~A}_{3}, \mathrm{~A}_{4}\right)$, cada uma com defasagem de $90^{\circ}$. A diferença de fase $\varphi$ entre o sinal emitido e o recebido é proporcional à distância entre o alvo e a câmara. A diferença de fase do sinal pode ser calculada através da Equação (1).

$$
\varphi=\arctan \left(\frac{A_{1}-A_{3}}{A_{2}-A_{4}}\right)
$$

onde:

$\varphi$ - Diferença de fase entre o sinal emitido e o recebido;

$A_{1}, A_{2}, A_{3}, A_{4}$ - Pontos amostrais para modelar a FC.

Além do valor da diferença de fase entre os sinais, dois outros valores podem ser calculados, a amplitude $a$ e o valor de intensidade $b$ do sinal recebido, conforme as Equações (2) e (3), respectivamente.

$$
\begin{aligned}
& a=\frac{\sqrt{\left(A_{1}-A_{3}\right)^{2}+\left(A_{2}-A_{4}\right)^{2}}}{2} \\
& b=\frac{A_{1}+A_{2}+A_{3}+A_{4}}{4}
\end{aligned}
$$

onde:

$a$ - Amplitude do sinal recebido;

$b$ - Intensidade do sinal recebido (offset). 
Com isto a distância $d$ do sensor ao alvo pode ser calculada através da Equação (4).

$$
d=\frac{c \cdot \varphi}{4 \pi \cdot f_{\bmod }}
$$

onde:

$d$ - Distância entre o sensor e o alvo;

c - Valor constante da velocidade da luz;

$f_{\text {mod }}$ - Frequência da onda portadora do sinal modulado.

A frequência de modulação da onda portadora do sinal emitido estabelece a distância máxima que poderá ser medida para um determinado comprimento de onda. Em um sinal modulado com a frequência de $20 \mathrm{MHz}$ e comprimento de onda igual a 15 metros $\left(\lambda_{\text {mod }}=15 \mathrm{~m}\right)$, conforme a Equação (5), a distância que poderá ser medida é de 7,5 metros (MÖLLER et al., 2005).

$$
d_{\max }=\frac{\lambda_{\bmod }}{2}=7,5 m
$$

\subsection{Princípio de Funcionamento do Sensor PMD}

O dispositivo PMD realiza medidas de distâncias utilizando o princípio TOF. Para isto emite sinais luminosos modulados com a mesma frequência e o sensor capta a quantidade de energia recebida. Com isto, informações sobre a fase e a intensidade do sinal recebido podem ser obtidas com o processamento.

O sinal modulado é emitido com uma fase determinada pelo oscilador do sistema. Para que se possa realizar a medida da distância, espera-se que o sinal seja recebido com fase diferente do sinal emitido. No sensor PMD as cargas elétricas do sinal recebido são separadas de acordo com a tensão do diodo de leitura. $\mathrm{O}$ arquivo de saída deste dispositivo contém informação de fase e intensidade do sinal recebido, o que gera uma imagem de intensidade com a informação de distância dos píxeis.

A quantidade de carga captada pelos diodos de leitura depende da diferença de fase entre o sinal emitido e o recebido pelo sensor. Esta informação é utilizada para calcular a diferença de fase entre a onda emitida e a recebida, pois é diretamente proporcional à carga que cada diodo captou. No entanto, se a luz incidente for modulada por um sinal retangular, mas a diferença de fase entre o sinal emitido e o refletido for igual a zero, a carga será captada por apenas um dos fotodiodos e a distância calculada será igual a zero (MÖLLER et al., 2005).

Um modelo de câmara TOF que utiliza o sensor PMD é CamCube 2.0. Este equipamento funciona em ambientes internos e externos, com um sensor PhotonICs PMD41k-S de 204x204 pixels, com dimensão de $45 \mu \mathrm{m}$, que captam os níveis de cinza da imagem de intensidade. A distância focal nominal desta câmara é igual a $12,8 \mathrm{~mm}$. Têm campo de visão (Field Of View - FOV) de $40^{\circ} \times 40^{\circ}$ que é variável, 
possibilita a diminuição de distorções, manchas ou outras imperfeições na imagem. O software de interface da câmara PMD apresenta a possibilidade de trabalhar com dados brutos para o processamento das imagens, além de funcionar em diferentes canais de frequências. Disponibiliza interface com outros softwares tais como o Matlab. Imagens com informação de distância dos píxeis podem ser obtidas de 0,3 à 7metros (PMD Technologies, 2009).

\section{METODOLOGIA}

A metodologia aplicada consiste em primeiramente corrigir a geometria da imagem TOF com POI obtidos com a calibração da câmara através do método proposto por Zhang (2000). Este método utiliza um painel contendo um alvo impresso com quadrados pretos e brancos, como um tabuleiro de xadrez, que é fotografado variando ângulos e posições. Estes quadrados são utilizados como pontos de controle e as imagens obtidas servem para realizar a calibração. Os parâmetros determinados são a distância focal, as coordenadas do ponto principal e as principais distorções causadas pelo sistema de lentes da câmara.

O passo seguinte foi a criação de uma imagem com as bordas dos objetos intensificadas a partir da segmentação do arquivo de distâncias, utilizando para isso uma análise da variação local dos autovalores.

A imagem com bordas intensificadas foi segmentada utilizando-se o algoritmo de deslocamento pela média.

Finalmente, as superfícies segmentadas, que são as partes de interesse do objeto, puderam ser separadas através do conjunto de pontos tridimensionais que as descreve, sendo então possível modelar as superfícies do objeto a partir da nuvem de pontos obtida.

\subsection{Segmentação}

Na etapa de segmentação separou-se o conjunto de dados da imagem TOF, em subconjuntos de pontos tridimensionais que compõe superfícies planas. É assumido que estas superfícies são partes de um objeto a ser identificado. Porém, para entender o princípio deste método de segmentação, é necessário antes apresentar a maneira utilizada para descrever uma superfície plana no espaço tridimensional a partir de seus autovalores e autovetores.

\subsection{Análises de Superfícies Através de Autovalores e Autovetores}

Autovalores e autovetores podem ser calculados a partir da Matriz de Variâncias e Covariâncias $(M V C)$ de um conjunto de observações. O tamanho relativo e direção dos autovetores indicam propriedades locais de superfícies (PAULY et al., 2002).

A $M V C$ é obtida a partir das coordenadas dos $k$ pontos vizinhos mais próximos do ponto em questão sendo calculada através da Equação (6) (JOHNSON e WICHERN, 2002). 


$$
M V C_{3 x 3}=\frac{1}{k} \sum_{i=1}^{k}\left[\vec{r}_{i}-\overrightarrow{\vec{r}}\right] \cdot\left[\vec{r}_{i}-\overrightarrow{\vec{r}}\right]^{T}=\left[\begin{array}{ccc}
\sigma_{x}^{2} & \sigma_{x y} & \sigma_{x z} \\
\sigma_{x y} & \sigma_{y}^{2} & \sigma_{y z} \\
\sigma_{x z} & \sigma_{y z} & \sigma_{z}^{2}
\end{array}\right]
$$

onde:

$M V C$ - Matriz de Variâncias e Covariâncias;

$\vec{r}_{i}$ - Vetor com as coordenadas tridimensionais do ponto em questão;

$\overrightarrow{\vec{r}}$ - Vetor com as coordenadas do centróide da superfície;

$\sigma_{x}^{2}, \sigma_{y}^{2}, \sigma_{z}^{2}$ - Variâncias;

$\sigma_{x y}, \sigma_{x z}, \sigma_{y z}$ - Covariâncias;

$k$ - Número de pontos da amostra.

$\mathrm{O}$ vetor de coordenadas do ponto $i$ da amostra é denotado com o vetor $\vec{r}_{i}$ pela Expressão (7). A posição do centróide pode ser calculada através da Equação (8).

$$
\begin{aligned}
& \vec{r}_{i}=\left[\begin{array}{lll}
x_{i} & y_{i} & z_{i}
\end{array}\right]^{T} \\
& \overrightarrow{\vec{r}}=\frac{1}{k} \sum_{i=1}^{k} \vec{r}_{i}
\end{aligned}
$$

Uma matriz $A$ quadrada, simétrica e positiva-semidefinida pode ser decomposta em autovetores $\vec{e}$ e autovalores $\lambda$ de acordo com a Equação (9). Os autovalores são escalares associados aos respectivos autovetores (LEON, 1998).

$$
A \cdot e=\lambda \cdot e
$$

De forma similar, a $M V C$ pode ser decomposta em autovalores e autovetores, conforme a Equação (10). A solução é possível através da decomposição espectral, também conhecida por decomposição de Jordan. Maiores detalhes podem ser vistos em Johnson e Wichern (1998), Shakarji (1998) e Belton (2008).

$$
M V C=\sum_{i=0}^{2} \lambda_{i} \cdot e_{i} \cdot e_{i}^{T}
$$

onde:

$e_{i}$ - Vetor com os autovetores $\vec{e}_{i}$;

$\lambda_{i}$ - Matriz os autovalores $\lambda_{i}$.

Como a $M V C$ é simétrica e positiva-semidefinida, os autovalores são números reais e os autovetores formam uma base ortogonal que corresponde aos componentes principais do conjunto de dados observados.

Podem-se definir superfícies planas em um conjunto de pontos 3D analisandose o menor autovalor $\lambda_{3}$, pois o correspondente autovetor $e_{3}$ será ortogonal a esta superfície que é definida pelos autovetores $e_{1}$ e $e_{2}$. 
A segmentação dos dados tridimensionais da imagem TOF baseia-se na análise da variação local dos autovalores e autovetores. A hipótese básica nesta abordagem é que ao longo de uma superfície plana os autovalores devem apresentar valores similares. Considerando duas regiões adjacentes e coplanares, então o tamanho relativo de seus autovetores deve ser igual. Se uma destas regiões encontra-se em uma borda, os autovalores calculados serão diferentes. Nesta análise, os autovalores de uma região em torno do píxel na imagem são calculados e armazenados em três novas matrizes. A região a ser considerada é uma janela quadrada em torno de um píxel central, cuja dimensão pode ser escolhida. O resultado desta segmentação são regiões onde os autovalores são aproximadamente iguais, definem uma superfície plana separada das regiões de bordas.

Os autovalores calculados para cada píxel são normalizados e escalados na faixa de 0 a 255 , usando 8 bits. Assim, os valores podem ser representados como uma imagem digital, passível de ser analisada com softwares de processamento. A imagem produzida pode então ser segmentada utilizando o algoritmo de deslocamento pela média.

\subsection{Algoritmo de Deslocamento pela Média}

$\mathrm{O}$ algoritmo de deslocamento pela média (Mean Shift) pode ser utilizado no reconhecimento de padrões para a segmentação de imagens. A densidade de pontos vizinhos a um ponto central pode ser estimada com a média do conjunto de dados discretos observados. A análise espacial de atributos é utilizada neste contexto com o objetivo de extrair informações, para isto utiliza a vizinhança de um ponto na imagem. Com os atributos escolhidos pode-se encontrar o centro das regiões com as maiores densidades, estas representam características significativas das superfícies dos objetos presentes na imagem.

Em aplicações estatísticas tradicionais é necessário verificar se a condição de normalidade dos dados é satisfeita no caso dos testes paramétricos. Isto porque se pressupõe o conhecimento da distribuição de probabilidade destes dados. Em outras aplicações podem-se utilizar métodos estatísticos não-paramétricos, com menos restrições sobre o comportamento dos dados. O conceito de Kernel é empregado em métodos não-paramétricos de estimativa de propriedades. Esta função retorna valores reais dos cálculos, sua integração resulta na unidade, além de atuar na imagem como uma janela de convolução que busca características similares dos elementos analisados (MACHADO, 2006).

Imagens podem ser representadas na forma de matrizes, onde cada posição é ocupada por vetores (píxeis), com dimensão $p$, onde $p=1$ no caso de imagens em níveis de cinza, e $p \geq 2$ no caso de imagens multiespectrais. O posicionamento de cada píxel na matriz imagem está contido no domínio espacial (Spatial domain) enquanto o valor de intensidade faz parte do domínio de características (Range domain). Com isto, cada elemento da matriz de posicionamento da imagem contém uma característica no espaço com domínio de dimensão $d=p+2$. Deste modo, um Kernel multivariado pode ser definido como o produto de dois Kernels radialmente 
simétricos em um espaço euclidiano, calculado com um parâmetro de cada domínio através da Equação (11), expressa nesta forma por Comaniciu e Meer (2002).

$$
K_{h_{s} h_{r}}(x)=\frac{c}{h_{s}^{2} h_{r}^{p}} k\left(\left\|\frac{\mathbb{x}^{s}}{h_{s}}\right\|^{2}\right) k\left(\left\|\frac{\mathbb{x}^{r}}{h_{r}}\right\|^{2}\right)
$$

onde:

$\mathbb{X}^{S}$ - Corresponde ao domínio espacial, posição do píxel na imagem;

$\mathbb{x}^{r}$ - Corresponde ao domínio de características, nível de cinza do píxel;

$k(x)$ - Elementos da função calculados em ambos os domínios;

$h_{s}, h_{r}$ - Parâmetros das bandas de cada domínio do Kernel empregado;

$C$ - Constante de normalização entre as bandas.

Para a utilização do algoritmo de deslocamento pela média devem ser escolhidos os parâmetros $h_{s}, h_{r}$ e $M$. Os parâmetros $h_{s}$ e $h_{r}$ correspondem às bandas de cada domínio do Kernel empregado, e o parâmetro $M$ corresponde à quantidade mínima de píxeis que deverão apresentar os segmentos da imagem segmentada, determinando assim a resolução na estimação da densidade.

O software EDISON (Edge Detection and Image SegmentatiON System) foi desenvolvido para realizar a segmentação de imagens utilizando o algoritmo de deslocamento pela média (CHRISTOUDIAS et al., 2002).

\subsection{Projeção Ortogonal dos Pontos}

Para a obtenção das coordenadas tridimensionais dos pontos do arquivo de distâncias da imagem TOF necessita-se de sua projeção ortogonal, isto ocorre devido ao fato do equipamento utilizado calcular distâncias radiais dos objetos em um sistema de coordenadas que pode ser considerado esférico.

A projeção ortogonal é feita utilizando a colinearidade entre o ponto no espaço objeto, o Centro Perspectivo (CP) do sistema de lentes da câmara, e o respectivo ponto no espaço imagem, conforme ilustra a Figura 3.

A distância ortogonal $Z$ dos pontos da imagem ao objeto pode ser calculada através da Equação (12).

$$
Z=d_{r} \cdot \cos \left(\tan ^{-1}\left(\sqrt{\frac{x^{2}+y^{2}}{f}}\right)\right)
$$

onde:

$Z$ - Distância ortogonal do centro perspectivo (CP) ao ponto;

$d_{r}$ - Distância radial do CP ao ponto no sistema esférico da câmara;

$f$ - Distância focal da câmara;

$x, y$ - Coordenadas dos pontos no sistema referencial fotogramétrico projetado na imagem. 
Figura 3 - Modelo geométrico para projeção ortogonal dos pontos.

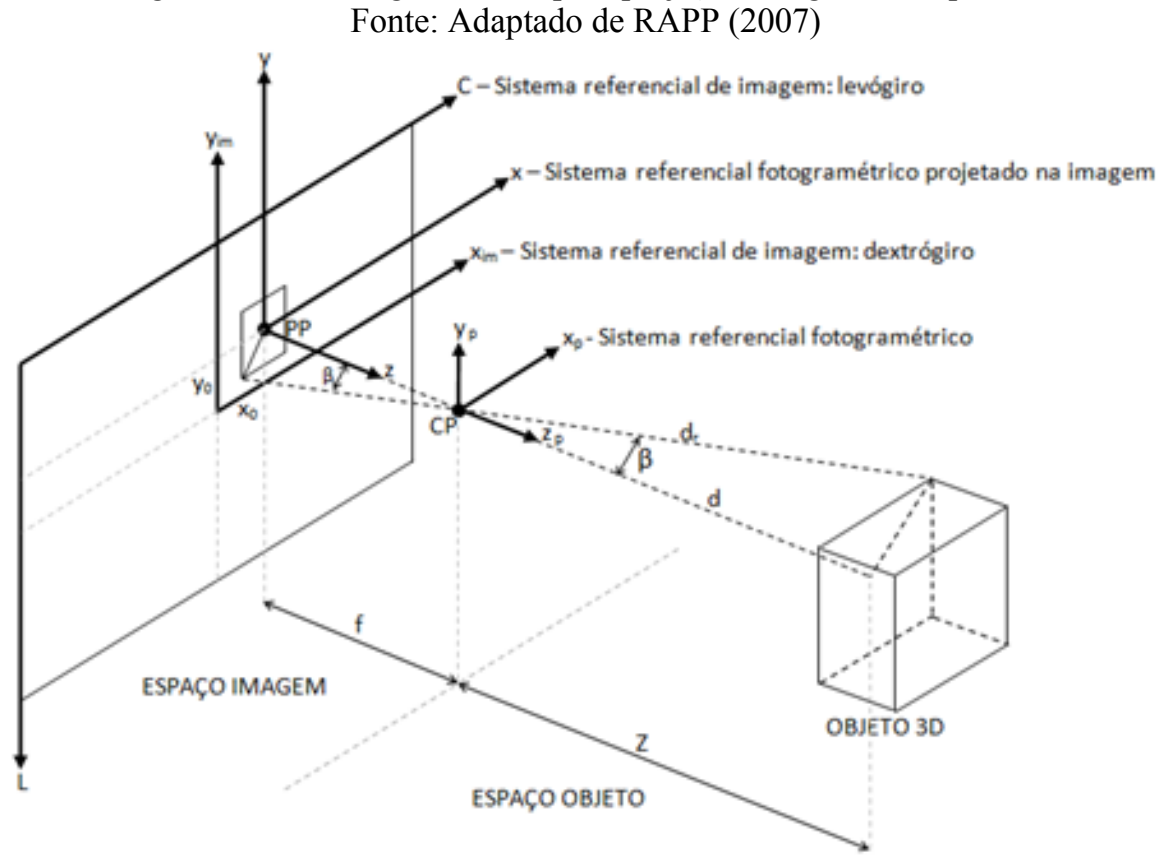

As coordenadas $X$ e $Y$ dos pontos no sistema referencial ortogonal, podem ser calculadas através das Equações (13) e (14).

$$
\begin{aligned}
& X=x \cdot \frac{z}{f_{x}} \\
& Y=y \cdot \frac{Z}{f_{y}}
\end{aligned}
$$

\section{DISCUSSÕES}

\subsection{Experimento: Segmentação de Superfícies a Partir da Imagem TOF}

Neste experimento o arquivo de distâncias da imagem TOF foi utilizado como ponto de partida para o cálculo dos autovalores locais das superfícies. A imagem foi obtida a uma distância aproximada de 1,3 m entre a câmara e o objeto de estudo, uma caixa com superfícies brancas e com padrão planar.

Primeiramente corrigiu-se a geometria dos dados utilizando os parâmetros obtidos com a calibração da câmara: distância focal calibrada $f_{c}$; coordenadas $x_{0} \mathrm{e}$ $y_{0}$ do ponto principal; e os coeficientes de distorção radial simétrica $k_{1}$ e $k_{2}$. 
O segundo passo foi criar uma imagem de intensidade com as bordas evidenciadas. Para isto, calcularam-se os autovalores locais para os três autovetores dos pontos do arquivo de distâncias da imagem TOF e geraram-se três bandas para a imagem nova, cada banda correspondendo a um dos autovalores calculados.

O terceiro passo foi segmentar a imagem de intensidade com as bordas evidenciadas do objeto através do algoritmo de deslocamento pela média. Com a imagem de intensidade segmentada pôde-se escolher as superfícies que teriam seus píxeis projetados para a nuvem de pontos $3 \mathrm{D}$.

A abordagem para a criação de uma imagem com bordas evidenciadas consiste em definir uma pequena vizinhança quadrada, uma janela móvel, e usar as coordenadas tridimensionais dos pontos nesta vizinhança para o cálculo dos autovalores, que após a normalização são armazenados na forma de uma imagem de intensidade.

O cálculo de autovalores para regiões em uma nuvem de pontos $3 \mathrm{D}$ possibilita colocar em evidência as bordas das superfícies do objeto que se pretende segmentar. A Figura 3 ilustra imagens com bordas intensificadas geradas utilizando autovalores

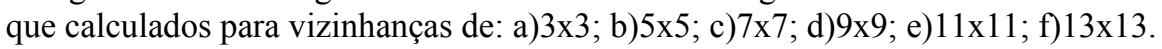

Figura 3 - Imagens de intensidade com bordas intensificadas.

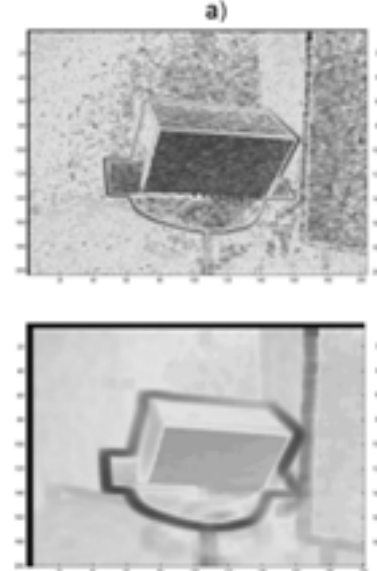

d)
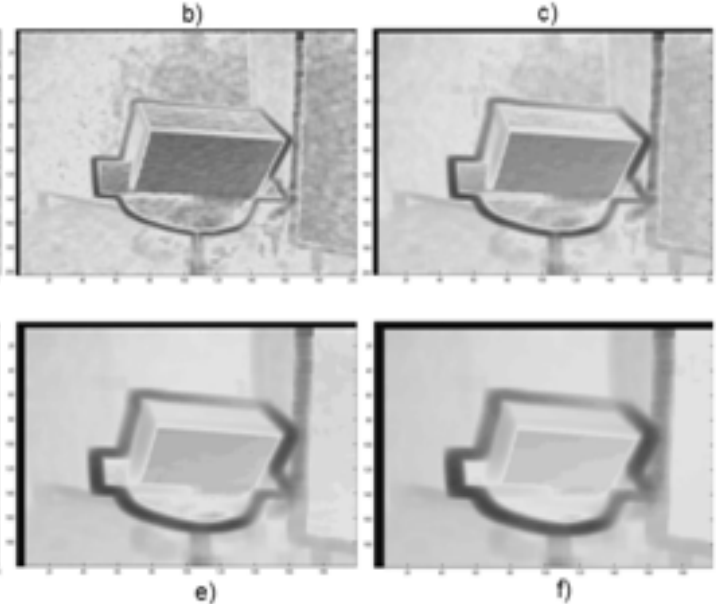

Com a Figura 3 é possível visualizar que se a quantidade de pontos utilizados no cálculo dos autovalores da região for grande, maior será a suavização sobre as superfícies e mais larga será a borda que limita os objetos presentes na cena. Para cada um destes arquivos geraram-se imagens de intensidade com três bandas correspondentes ao seu conjunto de autovalores $\lambda_{1}, \lambda_{2}$ e $\lambda_{3}$. Para este experimento utilizou-se a imagem ilustrada com a Figura $3 \mathrm{~b}$, calculada com o segundo autovalor 
$\lambda_{2}$. Esta imagem foi escolhida por apresentar linhas estreitas limitando as bordas do objeto e uniformidade nos valores digitais dos píxeis. Isto possibilitou manter a forma do objeto de estudo. Realizou-se a segmentação da imagem de intensidade com o algoritmo de deslocamento pela média. O resultado desta segmentação pode ser visto com a Figura 4.

Figura 4 - Segmentação da imagem de intensidade.
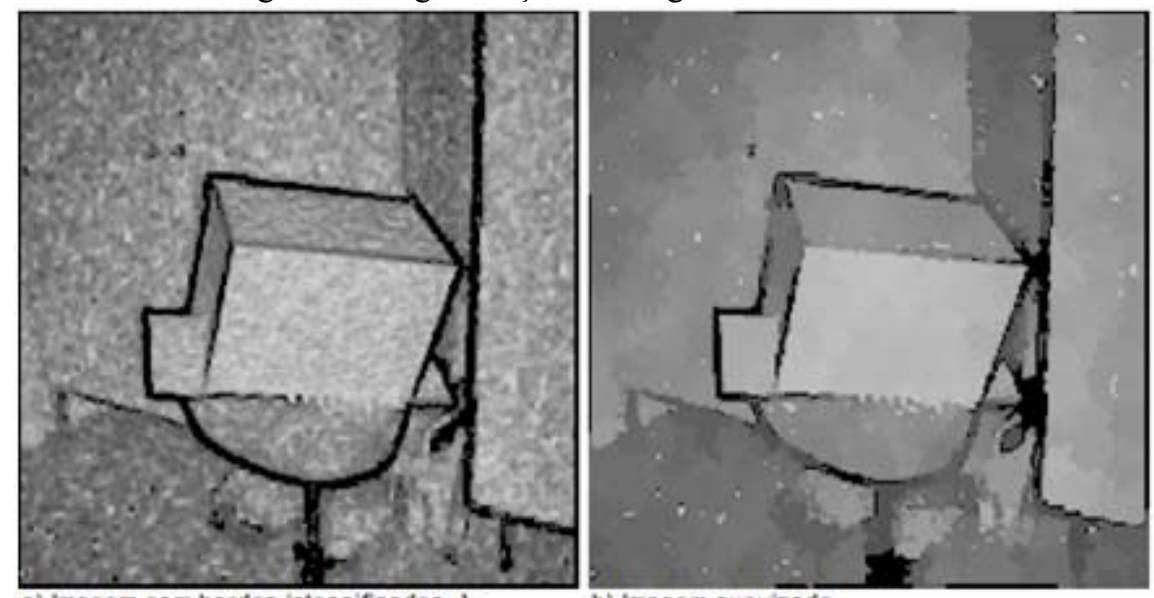

a) Imagem com bordas intensificadas, $\lambda$ :

b) Imagem suavizada
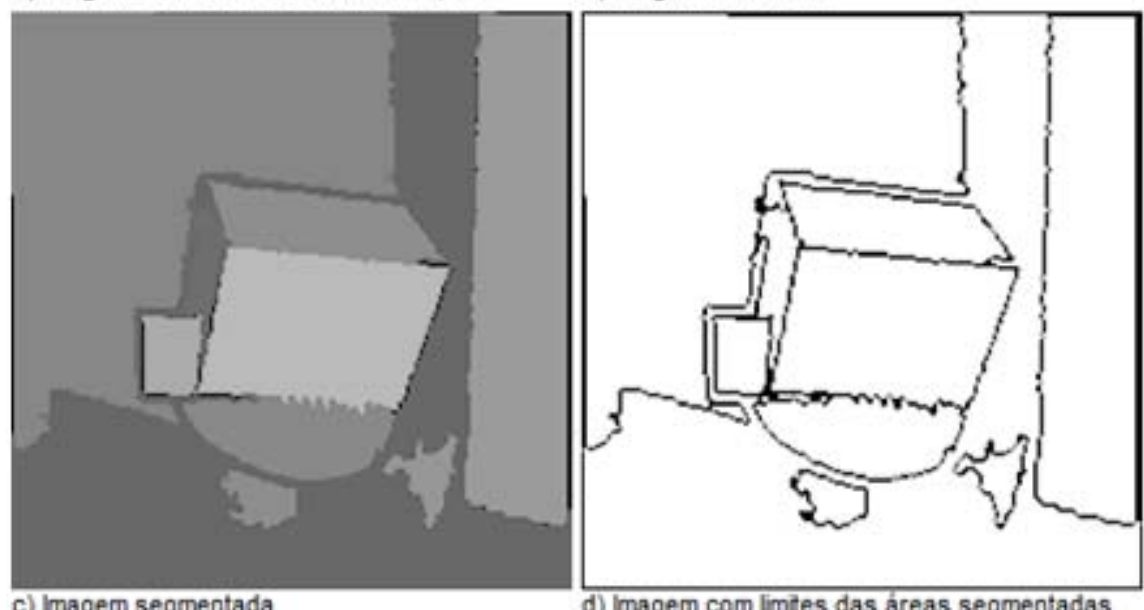

c) Imagem segmentada

d) Imagem com limites das áreas segmentadas

A Figura $4 \mathrm{a}$ corresponde à imagem com bordas intensificadas a ser segmentada, gerada com o segundo conjunto de autovalores. Na Figura $4 \mathrm{~b}$ é representada a imagem suavizada por filtragem. A imagem segmentada com o 
algoritmo de deslocamento pela média é ilustrada com a Figura 4c. Uma imagem com os píxeis que limitam as regiões segmentadas é ilustrada com a Figura $4 \mathrm{~d}$. A segmentação foi realizada com os parâmetros de cálculo $\left(h_{s}, h_{r}, M\right)=(7,9,300)$. Estes parâmetros correspondem ao domínio espacial da imagem $h_{s}$, domínio de características $h_{r}$ e tamanho da região mínima segmentada $M$. O parâmetro $M$ é fixado para evitar que regiões pequenas sejam incluídas na segmentação da imagem.

Com a imagem segmentada da Figura 4c, projetaram-se os pontos de interesse para a nuvem 3D. Isto foi feito utilizando as coordenadas dos píxeis na imagem de intensidade e seus correspondentes pontos no arquivo de distâncias da imagem TOF. Estes pontos foram escolhidos sobre as superfícies do objeto de estudo. A nuvem de pontos 3D resultante é apresentada com a Figura 5.

Figura 5 - Nuvem de pontos 3D gerada com a imagem segmentada.

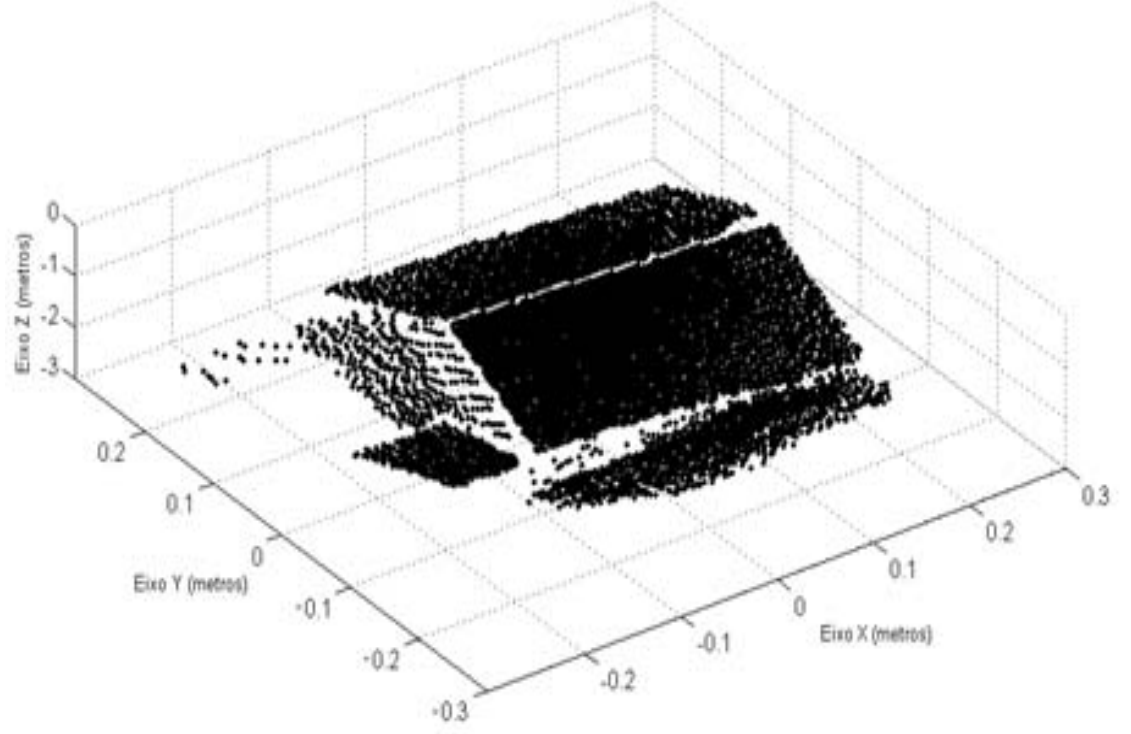

Escolheram-se quatro pontos extremos nas superfícies e calcularam-se as dimensões de três arestas do objeto para avaliar a exatidão obtida com a metodologia proposta. A Figura 6 apresenta a nuvem com os quatro pontos escolhidos nas superfícies do objeto. 
Figura 6 - Nuvem de pontos 3D dos segmentos.

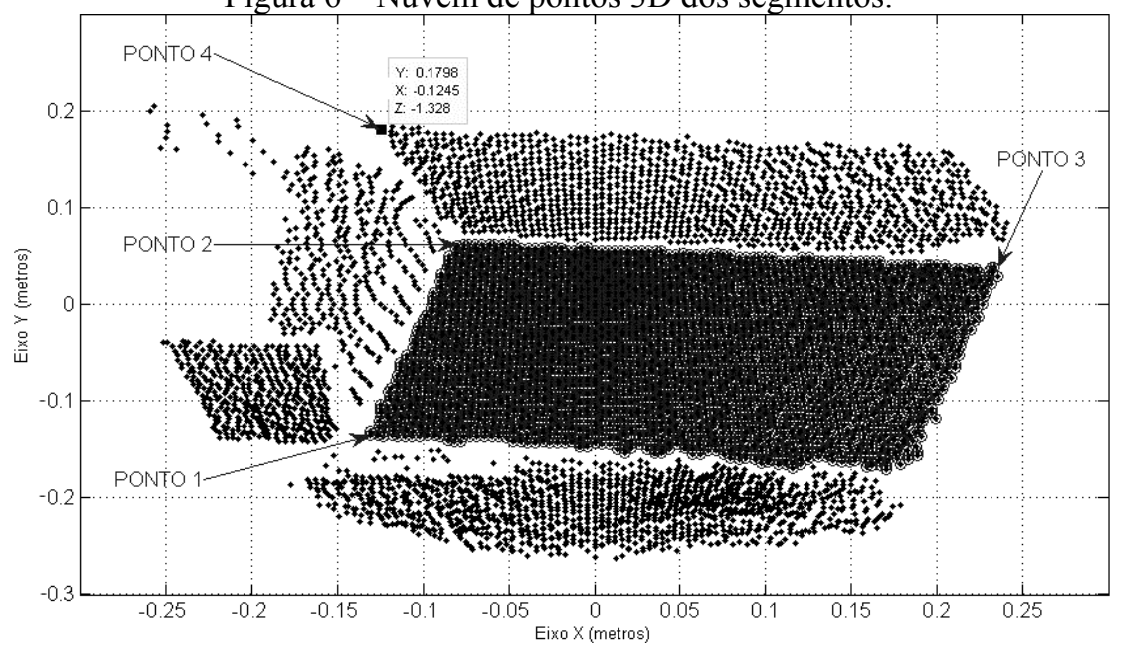

Com as coordenadas tridimensionais destes quatro pontos calcularam-se as dimensões de três arestas do objeto que foram comparadas com as medidas de referência ilustradas com a Figura 7.

Figura 7 - Dimensões de referência do objeto.

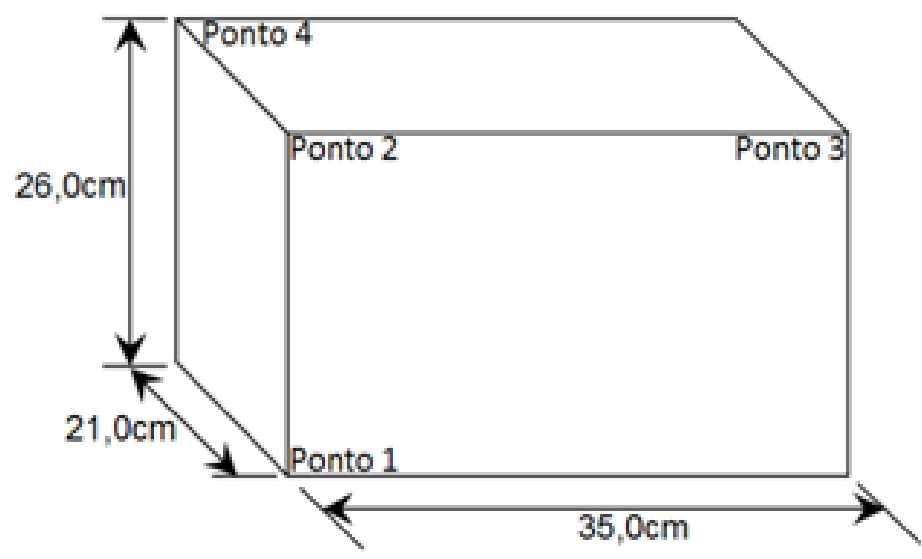

Sendo assim, a partir das coordenadas tridimensionais dos quatro pontos selecionados, obtiveram-se os resultados expostos com a Tabela 1. 
Tabela 1 - Coordenadas dos pontos lidos na nuvem 3D.

\begin{tabular}{c|c|c|c|c|c|c|c|c}
\hline & \multicolumn{3}{|c|}{ Coordenadas } & & \multicolumn{2}{c|}{ Medida realizada } & \multicolumn{2}{c}{ Diferenças } \\
\hline Ponto & $\mathrm{X}(\mathrm{cm})$ & $\mathrm{Y}(\mathrm{cm})$ & $\mathrm{Z}(\mathrm{cm})$ & Arestas & $\begin{array}{c}\text { Pontos } \\
\text { 3D }(\mathrm{cm})\end{array}$ & $\begin{array}{c}\text { Referência } \\
(\mathrm{cm})\end{array}$ & $(\mathrm{cm})$ & $(\%)$ \\
\hline 1 & $-13,1$ & $-13,6$ & $-121,9$ & Pontos: $1-2$ & 23,6 & 26,0 & 2,4 & 9,1 \\
\hline 2 & $-8,0$ & 6,1 & $-109,8$ & Pontos: $2-3$ & 33,2 & 35,0 & 1,8 & 5,2 \\
\hline 3 & 23,4 & 3,8 & $-120,3$ & Pontos: $2-4$ & 26,3 & 21,0 & 5,3 & 25,1 \\
\hline 4 & $-12,5$ & 17,9 & $-132,8$ & & & & & \\
\hline
\end{tabular}

Os melhores resultados foram obtidos com as medidas realizadas na aresta frontal localizada entre os pontos 2 e 3, que teve diferença em relação à medida de referência igual a $1,8 \mathrm{~cm}$, o que representa $5 \%$ desta distância. $\mathrm{O}$ pior resultado foi obtido com o cálculo da dimensão da aresta entre os pontos 2 e 4, com diferença de $5,3 \mathrm{~cm}$ o que significa $25 \%$ da medida de referência. Isto indica que os pontos projetados com maior profundidade para a nuvem $3 \mathrm{D}$, e com a superfície pouco aparente na imagem, tiveram maior erro no posicionamento tridimensional. Esta superfície encontrava-se bastante inclinada em relação ao plano da imagem, motivo pelo qual maiores diferenças de medida podem ser esperadas. Nos pontos 1, 2 e 3 , da superfície frontal, a diferença é menor, na ordem de 2 centímetros.

Realizou-se a modelagem tridimensional das superfícies frontal e a superior do objeto de estudo, conforme ilustra a Figura 8.

Figura 8 - Superfícies 3D modeladas do objeto de estudo.

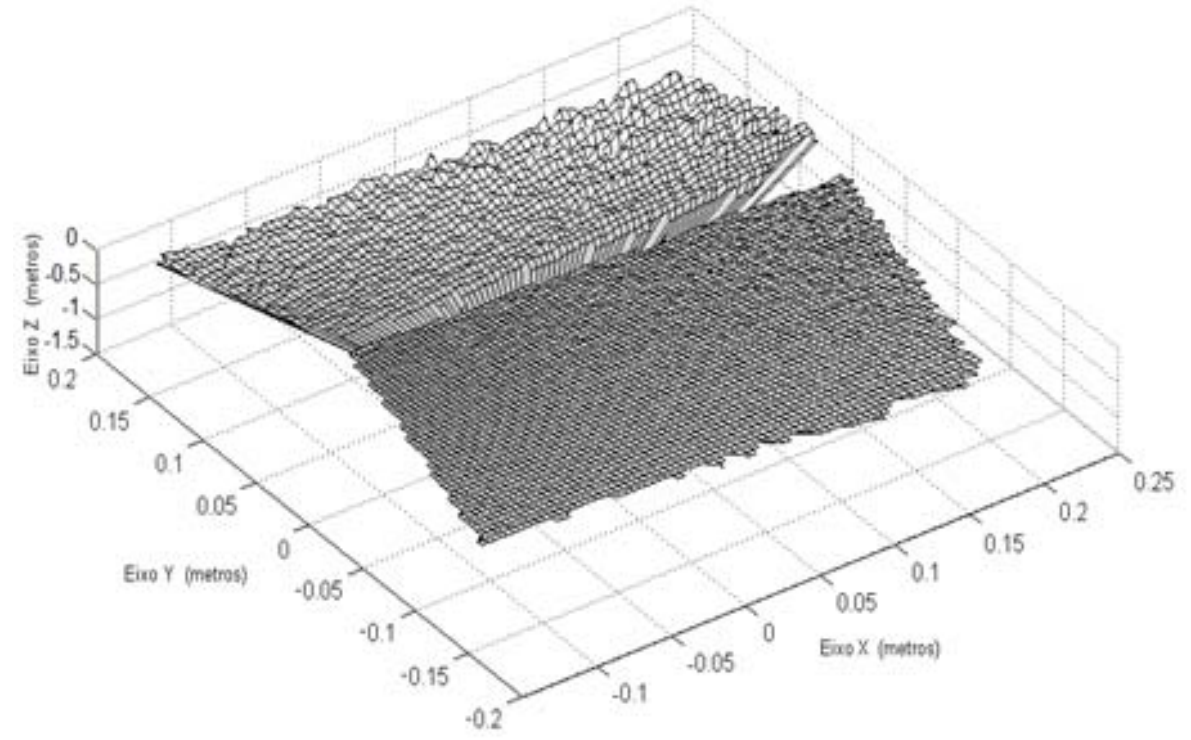

Bol. Ciênc. Geod., sec. Artigos, Curitiba, v. 17, nº 3, p.361-378, jul-set, 2011. 


\section{CONCLUSÃO}

A nuvem de pontos 3D gerada com as superfícies segmentadas utilizando a imagem TOF com bordas intensificadas mostrou-se satisfatória. No experimento realizado possibilitou a extração de informações métricas da nuvem de pontos 3D com diferença de dois centímetros em relação à medida de referência.

Foram encontradas medidas com maior exatidão quando a superfície do objeto estava paralela ao plano da imagem. Esta qualidade também é afetada pela distância entre a câmara e o objeto, pela refletividade das superfícies e pela iluminação ambiente da cena. A aresta frontal do objeto apresentou menor diferença de medida, 1,8 centímetros, que equivale a $5 \%$ da distância de referência medida.

Comparativamente à diferença de 2 centímetros encontrada entre a medida realizada com os pontos 3D e a medida de referência podem-se citar os seguintes trabalhos: Kahlmann et al. (2006) obtiveram diferenças de $1 \mathrm{~cm}$ nas medidas realizadas com os pontos 3D e a medida de referência, após realizar a calibração da câmara e com alvo à distância de 2,5m; Wiedemann et al. (2008) obtiveram diferenças na ordem de $4 \mathrm{~cm}$ após a calibração da câmara TOF e com um alvo medido a distância aproximada de $4 \mathrm{~m}$; May et al. (2008) obtiveram o valor de $10,5 \mathrm{~m}$ para a medida de $10,8 \mathrm{~m}$ considerada como referência, diferença de $30 \mathrm{~cm}$, sendo que as imagens TOF foram captadas do centro de uma sala, girando a câmara $360^{\circ}$; Kolb et al. (2009) obtiveram diferença de $2 \mathrm{~cm}$ para distâncias medidas de $1,0 \mathrm{~m}$ à $4,5 \mathrm{~m}$ utilizando imagens TOF obtidas com a câmara SR 400 e corrigidas geometricamente.

Com isto, conclui-se que as imagens TOF podem ser utilizadas para a obtenção de informações métricas de objetos a distâncias de até 7,0 metros. Com estas imagens podem-se gerar nuvens de pontos $3 \mathrm{D}$, segmentadas de acordo com as superfícies dos objetos na imagem. Os pontos utilizados na representação destas superfícies são selecionados na imagem de intensidade segmentada e projetados ortogonalmente para representar as superfícies de interesse.

Medidas com maior qualidade podem ser obtidas realizando uma correção radiométrica da imagem TOF em conjunto com a correção geométrica. A iluminação exterior ao sistema deve ser evitada, pois causa maior presença de ruído na imagem e, consequentemente, aumenta o erro no posicionamento dos pontos 3D.

\section{AGRADECIMENTOS}

Os autores deste artigo agradecem ao Programa de Pós-Graduação em Ciências Geodésicas Universidade Federal do Paraná (PPGCG/UFPR), pela infraestrutura disponibilizada para a realização deste trabalho; Ao Conselho Nacional de Desenvolvimento Científico e Tecnológico (CNPq) pelo fomento da pesquisa; Ao instituto de Fotogrametria e Sensoriamento Remoto da Universidade de Karlsruhe (KIT - Karlsruhe Institute of Tecnology) na Alemanha pela cooperação. 


\section{REFERÊNCIAS BIBLIOGRÁFICAS}

COMANICIU, D.; MEER, P. Mean Shift: A Robust Approach Toward Feature Space Analysis. 17p. IEEE Transactions on Pattern Analysis and Machine Intelligence, Vol. 24, N. 5. pp. 603-619, 2002.

CHRISTOUDIAS, M. C.; GEORGESCU, B.; MEER, P. Synergism in Low Level Vision. IEEE Transactions on Pattern Analysis and Machine Intelligence, Vol. 24, No. 5, pp. 603-619, 2002.

JAMTSHO, S. Geometric modelling of 3D Range Cameras and their application for structural deformation measurement. 174p. (Master's Thesis, Department of Geomatics Engineering). University of Calgary, Alberta, 2010.

JOHNSON, R. A.; WICHERN, D. W. Applied Multivariate Statistical Analysis. 4. ed. New Jersey: Pearson Prentice Hall, 1998.

KAHLMANN, T.; REMONDINO, F.; INGENSAND, H. Calibration for increased accuracy of the range imaging camera Swissranger ${ }^{T M}$. ISPRS Commission V Symposium 'Image Engineering and Vision Metrology', Vol. XXXVI, Part 5, 2006.

KOCH, R.; SCHILLER, I.; BARTCZAK, B.; KELLNER, F.; KÖSER, K. MixIn3D: $3 D$ Mixed Reality with ToF-Camera. 16p. In: Work Programme of the EU's $7^{\text {th }}$, Framework program, 2009.

KOLB, A.; BARTH, E.; KOCH, R.; LARSEN, R. Time-of-Flight Sensors in Computer Graphics. 16p. In: STAR - State of The Art Report. The Eurographics Association, 2009.

LANGE, R. 3D Time-of-Flight distance measurement with custom solid-state image sensors in CMOS/CCD-technology. $205 \mathrm{p}$. (Dissertation submitted to the Department of Electrical Engineering and Computer Science at University of Siegen for the degree of Doctor of Technical Sciences). Univesity of Siegen, Alemanha, 2000.

LEON, S. J. Linear Algebra with applications. 4. ed. Dartmouth: Prentice Hall, 1998. 390p.

MACHADO, A. M. L. Extração automática de contornos de edificações utilizando imagem gerada por câmara digital de pequeno formato e dados LIDAR. 201 p. Tese de doutorado apresentada ao Curso de Pós-Graduação em Ciências Geodésicas da Universidade Federal do Paraná. Curitiba: UFPR, 2006.

MAY, S.; DROESCHEL, D.; HOLTZ, D.; WIESEN, C. FUCHS, S. 3D Pose estimation and mapping with time-of-flight cameras. 6p. Germany, 2008.

MÖLlER, T.; KRAFT, H.; FREY, J.; ALBRECHT, M.; LANGE, R. Robust 3D Measuremente with PMD Sensors. 2005.

PAULY, M.; GROSS, M.; KOBBELT, L. P. Efficient simplification os pointsampled surfaces. 8p. In: VIS '02: Proceedings of the Conference on Visualization '02, Washington, DC, USA, 2002. IEEE Computer Society.

PMD Technologies. PMD [vison] ${ }^{\circledR}$ CamCube 2.0. Datasheet V. No. 20090601. 4p. Siegen, 2009. Disponível em: $<$ http://www.pmdtec.com>. 
RAPP, H. Experimental and Theoretical Investigation of Correlating TOF-Camera Systems. 71p. (Diploma Thesis in Physics, Faculty for Physics and Astronomy). University of Heidelberg, Alemanha, 2007.

RINGBECK, T. A 3D time of flight camera for object detection. 10p. In: Optical 3D Measurement Techniques, Plenary Session 1: Range Imaging I. Zürich, 2007.

SHAKARJI, C. M. Least-Squares fitting algorithms of the NIST algorithm testing system. 9p. Journal of research of the national of standards and technology, $\mathrm{v}$. 103, n. 6, 1998.

WIEDEMANN, M.; SAUER, M.; DRIEWER, F.; SCHILlING, K. Analysis and characterization of the PMD camera for application in mobile robotics. $6 \mathrm{p}$. In: Proceedings of the $17^{\text {th }}$ World Congress, The International Federation of Automatic Control. Seoul, Korea, July 6-11, 2008.

ZHANG, Z. A flexible new technique for camera calibration. 5p. In: IEEE Transactions on Pattern Analysis and Machine Intelligence, November, 2000.

Recebido em fevereiro de 2011. Aceito em julho de 2011.) 